PROCEEDINGS OF THE

AMERICAN MATHEMATICAL SOCIETY

Volume 130, Number 7, Pages 1877-1880

S 0002-9939(02)06471-7

Article electronically published on February 4, 2002

\title{
MAXIMAL BETTI NUMBERS
}

\author{
MARC CHARDIN, VESSELIN GASHAROV, AND IRENA PEEVA \\ (Communicated by Michael Stillman)
}

\begin{abstract}
We provide a short proof that the lexicographic ideal has the greatest Betti numbers among all graded ideals with a fixed Hilbert function.
\end{abstract}

\section{INTRODUCTION}

This paper provides a short proof that the Betti numbers of the lexicographic ideal are greatest among the Betti numbers of all homogeneous ideals with the same Hilbert function over a polynomial ring or over an exterior algebra. No step in the proof is new; we have just put together and simplified some arguments from AHH, $\mathrm{Bi}, \mathrm{Gr}, \mathrm{Hu}$. The proof has three ingredients: a well-known reduction to strongly stable ideals, Green's Theorem 1.2, and a formula for the Betti numbers given in Lemma 2.1 (or 3.1).

Let $k$ be a field. We will work over a ring $A$, that will be either the polynomial ring $S=k\left[x_{1}, \ldots, x_{n}\right]$ with char $(k)=0$, or the exterior algebra $E$ on $n$ variables $x_{1}, \ldots, x_{n}$ over $k$. The ring $A$ is graded by $\operatorname{deg}\left(x_{i}\right)=1$ for all $i$. Let $M$ be a monomial ideal in $A$. Denote by $\mathrm{G}(M)$ the unique set of minimal monomial generators of $M$. We say that $M$ is strongly stable if $x_{i} m \in M$ implies that $x_{p} m \in$ $M$ for $1 \leq p \leq i$. A monomial ideal $L$ is called lexicographic if for every $j \in \mathbf{N}$ the space $L_{j}$ is spanned by the first $\operatorname{dim}\left(L_{j}\right)$ monomials in the lexicographic order. Every lexicographic ideal is strongly stable.

Throughout, $J$ stands for a homogeneous ideal in $A$. Its graded Betti numbers $\beta_{i, i+j}^{A}(J)$ are bounded above by those of any initial ideal in $(J)$; cf. e.g. $\overline{G r}$ Corollary 1.21]. A generic initial ideal is strongly stable; cf. e.g. Gr. Proposition 1.2 and Theorem 1.27]. Thus, there exists a strongly stable ideal $I$ with the same Hilbert function as $J$ such that

$$
\beta_{i, i+j}^{A}(J) \leq \beta_{i, i+j}^{A}(I) \quad \text { for all } i, j .
$$

By Macaulay's Theorem and Kruskal-Katona's Theorem there exists a lexicographic ideal $L$ with the same Hilbert function as $I$; simpler (than the original ones) proofs of these theorems are given in $\mathrm{Gr}$, Theorem 3.3, Proposition 3.7, Theorem 5.1]. Note that $\left|\mathrm{G}(J)_{j}\right| \leq\left|\mathrm{G}(I)_{j}\right| \leq\left|\mathrm{G}(L)_{j}\right|$; this was extended to all graded Betti numbers in $\mathrm{AHH}, \mathrm{Bi}, \mathrm{Hu}$ as follows.

Received by the editors June 1, 2000

2000 Mathematics Subject Classification. Primary 13D02.

(C)2002 American Mathematical Society 
Theorem 1.1. Let $J$ be a homogeneous ideal in $A$. If $L$ is the lexicographic ideal with the same Hilbert function as $J$, then

$$
\beta_{i, i+j}^{A}(J) \leq \beta_{i, i+j}^{A}(L) \quad \text { for all } i, j .
$$

We prove this theorem. The above discussion shows that it suffices to establish the inequalities

$$
\beta_{i, i+j}^{A}(I) \leq \beta_{i, i+j}^{A}(L) .
$$

For a monomial $m$ we set $\max (m)=\max \left\{i \mid x_{i}\right.$ divides $\left.m\right\}$, and for a monomial ideal $M$ we denote by $M_{j}^{\#}$ the set of all monomials in $M_{j}$. Furthermore, for a set of monomials $\mathcal{M}$ let

$$
w_{p}(\mathcal{M})=|\{m \in \mathcal{M} \mid \max (m)=p\}|
$$

and

$$
w_{\leq p}(\mathcal{M})=|\{m \in \mathcal{M} \mid \max (m) \leq p\}|
$$

In particular, $w_{\leq n}\left(M_{j}^{\#}\right)=\operatorname{dim}\left(M_{j}\right)$. We will use the following result, which is equivalent to Green's Theorem $\mathrm{Gr}$, Theorems 3.4 and 5.2]:

Theorem 1.2. If I is strongly stable and $L$ is the lexicographic ideal with the same Hilbert function as $I$, then

$$
w_{\leq p}\left(L_{j}^{\#}\right) \leq w_{\leq p}\left(I_{j}^{\#}\right) \quad \text { for all } p, j .
$$

Remark. Green's Theorem [Gr, Theorems 3.4 and 5.2] is stated for a homogeneous ideal and a generic linear form. Replacing the ideal by a generic initial ideal, we reduce to the strongly stable case. In this case $x_{n}$ is a generic linear form. Also note that $w_{\leq n-1}\left(I_{j}^{\#}\right)=\operatorname{dim}\left(I_{j} / I_{j} \cap\left(x_{n}\right)\right)$.

\section{Proof of Theorem 1.1 over a Polynomial Ring}

$$
\text { (I.E. IN THE CASE } A=S \text { ) }
$$

Lemma 2.1. If $I$ is strongly stable in $S$, then

$$
\beta_{i, i+j}^{S}(I)=\left|I_{j}^{\#}\right|\left(\begin{array}{c}
n-1 \\
i
\end{array}\right)-\sum_{p=1}^{n-1} w_{\leq p}\left(I_{j}^{\#}\right)\left(\begin{array}{c}
p-1 \\
i-1
\end{array}\right)-\sum_{p=1}^{n} w_{\leq p}\left(I_{j-1}^{\#}\right)\left(\begin{array}{c}
p-1 \\
i
\end{array}\right) .
$$

Proof. The Eliahou-Kervaire minimal free resolution of $I$ (see [EK]) has basis

$$
\left\{\left(m ; t_{1}, \ldots, t_{i}\right) \mid m \in \mathrm{G}(I), 1 \leq t_{1}<\cdots<t_{i}<\max (m) \text { natural numbers }\right\},
$$

where the element $\left(m ; t_{1}, \ldots, t_{i}\right)$ has homological degree $i$ and internal degree $i+$ $\operatorname{deg}(m)$. Hence, the Betti numbers of $I$ are

$$
\beta_{i, i+j}^{S}(I)=\sum_{m \in \mathrm{G}(I)_{j}}\left(\begin{array}{c}
\max (m)-1 \\
i
\end{array}\right)=\sum_{p=1}^{n} w_{p}\left(\mathrm{G}(I)_{j}\right)\left(\begin{array}{c}
p-1 \\
i
\end{array}\right) .
$$

Now we perform a short computation introduced by Bigatti in [Bi]: We have

$$
\mathrm{G}(I)_{j}=I_{j}^{\#} \backslash I_{j-1}^{\#} \cdot\left\{x_{1}, \ldots, x_{n}\right\} .
$$


Furthermore, since $I$ is strongly stable we have

$$
I_{j-1}^{\#} \cdot\left\{x_{1}, \ldots, x_{n}\right\}=\coprod_{p=1}^{n}\left\{x_{p}\right\} \cdot\left\{m \in I_{j-1}^{\#} \mid \max (m) \leq p\right\} .
$$

Therefore,

$$
\begin{aligned}
\beta_{i, i+j}^{S}(I) & =\sum_{p=1}^{n} w_{p}\left(I_{j}^{\#}\right)\left(\begin{array}{c}
p-1 \\
i
\end{array}\right)-\sum_{p=1}^{n} w_{\leq p}\left(I_{j-1}^{\#}\right)\left(\begin{array}{c}
p-1 \\
i
\end{array}\right) \\
& =\sum_{p=1}^{n}\left(w_{\leq p}\left(I_{j}^{\#}\right)-w_{\leq p-1}\left(I_{j}^{\#}\right)\right)\left(\begin{array}{c}
p-1 \\
i
\end{array}\right)-\sum_{p=1}^{n} w_{\leq p}\left(I_{j-1}^{\#}\right)\left(\begin{array}{c}
p-1 \\
i
\end{array}\right) \\
& =\left|I_{j}^{\#}\right|\left(\begin{array}{c}
n-1 \\
i
\end{array}\right)-\sum_{p=1}^{n-1} w_{\leq p}\left(I_{j}^{\#}\right)\left(\begin{array}{c}
p-1 \\
i-1
\end{array}\right)-\sum_{p=1}^{n} w_{\leq p}\left(I_{j-1}^{\#}\right)\left(\begin{array}{c}
p-1 \\
i
\end{array}\right) .
\end{aligned}
$$

Proof of Theorem 1.1 over a polynomial ring. Both $I$ and $L$ are strongly stable ideals. Use the formula for the Betti numbers in Lemma 2.1 and apply Green's Theorem 1.2.

\section{Proof of Theorem 1.1 over AN exterior Algebra}

(I.E. IN THE CASE $A=E$ )

If $M$ is a monomial ideal over $E$ generated by square-free monomials $m_{1}, \ldots, m_{r}$, then we denote by $\tilde{M}$ the ideal in $S$ generated by $m_{1}, \ldots, m_{r}$. Thus, we have Betti numbers $\beta_{i, i+j}^{E}(M)$ of $M$ over $E$ and also Betti numbers $\beta_{i, i+j}^{S}(\tilde{M})$ of $\tilde{M}$ over $S$.

Lemma 3.1. If I is strongly stable in $E$, then

$$
\beta_{i, i+j}^{S}(\tilde{I})=\left|I_{j}^{\#}\right|\left(\begin{array}{c}
n-j \\
i
\end{array}\right)-\sum_{p=1}^{n-1} w_{\leq p}\left(I_{j}^{\#}\right)\left(\begin{array}{c}
p-j \\
i-1
\end{array}\right)-\sum_{p=1}^{n} w_{\leq p-1}\left(I_{j-1}^{\#}\right)\left(\begin{array}{c}
p-j \\
i
\end{array}\right) .
$$

Note that although the Betti numbers are over $S$, we use the invariants $w_{p}$ and $w_{\leq p}$ over $E$.

Proof. Denote by $M$ the smallest (with respect to inclusion) strongly stable ideal in

$S$ containing $\tilde{I}$. Let $\mathbf{F}_{M}$ be the Eliahou-Kervaire resolution of $M$. This resolution is $\mathbf{N}^{n}$-graded and can be written as $\mathbf{F}_{M}=\bigoplus_{i \geq 0, \mathbf{a} \in \mathbf{N}^{n}} F_{i, \mathbf{a}}$, where $F_{i, \mathbf{a}}$ is a free $S$ module generated in $\mathbf{N}^{n}$-degree $\mathbf{a}$ and homological degree $i$. Consider the truncation $\mathbf{F}=\bigoplus_{i \geq 0, \mathbf{a} \in(0,1)^{n}} F_{i, \mathbf{a}}$, called the square-free part of $\mathbf{F}_{M}$. The complex $\mathbf{F}$ is exact in square-free degrees since it coincides with $\mathbf{F}_{M}$ in such degrees. Taylor's resolution shows that the Betti numbers of $\tilde{I}$ vanish in non-square-free degrees. Hence, $\mathbf{F}$ is the minimal free resolution of $\tilde{I}$ over $S$. Therefore, by (2.2) the minimal free resolution of $\tilde{I}$ has basis $\left\{\left(m ; t_{1}, \ldots, t_{i}\right) \mid m \in \mathrm{G}(I), 1 \leq t_{1}<\cdots<t_{i}<\right.$ $\max (m), m x_{t_{1}} \ldots x_{t_{i}}$ is square-free $\}$, so we have the Betti numbers

$$
\beta_{i, i+j}^{S}(\tilde{I})=\sum_{p=1}^{n} w_{p}\left(\mathrm{G}(I)_{j}\right)\left(\begin{array}{c}
p-j \\
i
\end{array}\right)
$$

Since $I$ is strongly stable, it follows that (in $E$ ) we have $I_{j-1}^{\#} \cdot\left\{x_{1}, \ldots, x_{n}\right\}=$ $\coprod_{p=1}^{n}\left\{x_{p}\right\} \cdot\left\{m \in I_{j-1}^{\#} \mid \max (m)<p\right\}$. Therefore, a minor modification of the computation in the proof of Lemma 2.1 provides the desired formula. 
Proof of Theorem 1.1 over an exterior algebra. Both $I$ and $L$ are strongly stable ideals. Using the formula for the Betti numbers in Lemma 3.1 and applying Green's Theorem 1.2 we get

$$
\beta_{i, i+j}^{S}(\tilde{I}) \leq \beta_{i, i+j}^{S}(\tilde{L}) \text { for all } i, j ;
$$

this was first proved in [AHH, Theorem 4.4].

For any monomial ideal $N$ in $E$ we have the following relation between the Betti numbers over $S$ and those over $E$ :

$$
\sum_{i, j} \beta_{i, i+j}^{E}(E / N) t^{i} v^{i+j}=\sum_{i, j} \beta_{i, i+j}^{S}(S / \tilde{N}) t^{i} v^{i+j} \frac{1}{(1-t v)^{j}}
$$

this was first proved in [AAH, Proposition 2.1], later a simpler proof was given in EPY]. Combining the above formula and (3.2) provides the desired inequalities.

\section{REFERENCES}

[Bi] A. Bigatti: Upper bounds for the Betti numbers of a given Hilbert function, Comm. Algebra 21 (1993), 2317-2334. MR 94c:13014

[AAH] A. Aramova, L. Avramov, and J. Herzog: Resolutions of monomial ideals and cohomology over exterior algebras, Trans. Amer. Math. Soc. 352 (2000), 579-594. MR 2000c:13021]

[AHH] A. Aramova, J. Herzog, and T. Hibi: Squarefree lexsegment ideals, Math. Z. 228 (1998), 353-378. MR 99h:13013

[EPY] D. Eisenbud, S. Popescu, and S. Yuzvinsky: Hyperplane arrangements cohomology and monomials in the exterior algebra, Trans. Amer. Math. Soc., to appear.

[EK] S. Eliahou and M. Kervaire: Minimal resolutions of some monomial ideals, J. Algebra 129 (1990), 1-25. MR 91b:13019

[Gr] M. Green: Generic initial ideals, in Six lectures on commutative algebra, Birkhäuser, Progress in Mathematics 166, (1998), 119-185. MR 99m:13040

[Hu] H. Hulett: Maximum Betti numbers of homogeneous ideals with a given Hilbert function, Comm. Algebra 21 (1993), 2335-2350. MR 94c:13015

Institut de Mathématiques, UMR 7586 du CNRS, Université Pierre et Marie Curie, F-75252 Paris Cedex 05, France

Department of Mathematics, Cornell University, Ithaca, New York 14850

Department of Mathematics, Cornell University, Ithaca, New York 14850

Current address: Department of Mathematics, Purdue University, West Lafayette, Indiana 47907-1395 